REGARDS

SUR L'ECONOMIE ALLEMAND

BULLETIN ECONOMIQUE DU CIRAC
Regards sur l'économie allemande

Bulletin économique du CIRAC

$78 \mid 2006$

Varia

\title{
Une santé (presque) retrouvée
}

Isabelle Bourgeois

\section{CpenEdition}

Journals

Édition électronique

URL : http://journals.openedition.org/rea/742

DOI : 10.4000/rea.742

ISBN : 978-2-8218-0852-2

ISSN : 1965-0787

Éditeur

CIRAC

Édition imprimée

Date de publication : 1 octobre 2006

Pagination : 3-4

ISSN : 1156-8992

Référence électronique

Isabelle Bourgeois, «Une santé (presque) retrouvée », Regards sur l'économie allemande [En ligne], 78 | octobre 2006, mis en ligne le 05 mai 2008, consulté le 15 septembre 2020. URL : http://

journals.openedition.org/rea/742 


\section{Une santé (presque) retrouvée}

L'économie allemande tourne à plein régime. Pour la première fois depuis 2001 , la croissance repose sur une large assise. Si les exportations en restent le traditionnel moteur, la demande intérieure le relaie désormais : la reprise des investissements se confirme, et la consommation des ménages est en hausse. Cette dynamique commence à se répercuter sur le marché de l'emploi.

Depuis cet été, les économistes revoient à la hausse leurs prévisions de croissance pour l'année en cours : alors qu'elles s'échelonnaient entre $+1,6 \%$ (gouvernement fédéral) et $+2,1 \%$ (IfW), elles vont maintenant de $+2,1 \%$ (HWWA) à $+2,4 \%$ (IfW). Le rapport conjoint des instituts qui sera publié le 16 octobre s'inscrira dans cette fourchette. La santé retrouvée de l'économie allemande incite les experts à ne pas attendre d'effets foncièrement restrictifs de la hausse de la TVA (de $16 \%$ à $19 \%$ ) au $1^{\mathrm{er}}$ janvier. Tout au plus, elle tassera passagèrement la croissance de 2007 (+1,5\% probablement), mais ne remettra pas fondamentalement en cause la dynamique de l'économie, comme l'explique par exemple l'OCDE. La majorité des observateurs estime que, structurellement, le ralentissement attendu sur les marchés mondial et européen jouera un rôle plus important.

Selon Destatis, l'embellie s'est déclenchée à la fin de l'an dernier. Après $+0,3 \%$ au dernier trimestre 2005 , le PIB est en nette progression : $+0,7 \%$ au premier trimestre 2006 , puis $+0,9 \%$ (mais $+2,4 \%$ par rapport à avril-juin 2005). C'est là le meilleur taux enregistré depuis cinq ans. Au cours du premier semestre, le PIB allemand a été en hausse de $+2 \%$. Pour la première fois depuis longtemps, la croissance réelle a ainsi dépassé la croissance potentielle, estimée traditionnellement à un peu plus de $1 \%$.

En tête des facteurs conjoncturels à l'origine de cette embellie, la forte demande mondiale qui soutient le dynamisme d'une industrie qui fait dès lors office de "charnière entre un marché mondial en plein boom et les facteurs endogènes de croissance », comme le formule Michael Hüther, président de l'institut IW de Cologne (conférence de presse du 9 octobre). Le titre de championne du monde à l'export dynamise ainsi l'activité au plan intérieur. Certes, l'excédent commercial tend à se réduire, l'appréciation de l'euro par rapport au dollar et la flambée des prix pétroliers renchérissant les importations ; il n'est plus que de 1 point de pourcentage. Plus fondamentale est cependant la hausse continue des échanges : $+10 \%$ sur l'année pour les exportations, $+9 \%$ pour les importations (IW) ; cela traduit le bon positionnement de l'industrie allemande dans le monde. De ce fait, pour l'industrie, le principal risque conjoncturel réside moins dans la crise au Moyen Orient et son impact sur la facture énergétique que dans la « propension au protectionnisme » qui s'esquisse depuis l'échec des négociations de l'OMC (Doha).

En un an, la production industrielle a crû de $+5,0$ \%. Les secteurs phares se distinguent : la construction automobile et la construction mécanique. Cette dernière devrait battre tous les records cette année : les exportations ont crû de $+10,3 \%$ au premier semestre et, «pour la première fois, la production devrait dépasser en valeur les 155 milliards $€$ cette année », se réjouit Dieter Brucklacher, président de la fédération VDMA (Die Welt, 07-09-06). II fonde son optimisme sur une demande intérieure en plein boom depuis novembre 2005 ; de janvier à août, les entrées en commande nationales (+19\%) ont dépassé les commandes étrangères (+15\%).

Cette demande intérieure reflète la reprise de l'investissement depuis la fin de l'année. La tendance s'est nettement raffermie depuis : les entreprises, qui avaient d'abord accru le taux d'utilisation de leurs capacités, passent à l'extension de leurs capacités. Globalement, les investissements bruts ont ainsi augmenté de $+3,6 \%$ au second trimestre (Destatis). Mais, et cela est nouveau : si les investissements en biens d'équipement ont crû de $+2,0 \%$, c'est la construction qui s'est révélée la plus dynamique, avec $+5,2 \%$.

Cette évolution explique l'optimisme du BTP. «Pour la première fois depuis 1999, nous nous attendons cette année à une croissance du chiffre d'affaires », affirme Michael Knipper, directeur général de la fédération du bâtiment HDB (Handelsblatt, 24-08-06). Les données de Destatis confirment la fin de la crise de restructuration que traversait ce secteur que l'unification avait hypertrophié : il a enregistré une croissance de $+0,5 \%$ au premier trimestre 2006 comme au second. De janvier à juin, les entrées en commande ont augmenté de $8,6 \%$ selon la fédération. Toutes les activités sont concernées: les installations industrielles, tirées par les perspectives de rentabilité des entreprises et l'extension de leurs capacités principalement en Europe de l'est, mais aussi les travaux publics, dopés par l'amélioration de la situation budgétaire des communes. L'habitat, pour
Une croissance en nette hausse :...

$+2,0 \%$ au premier semestre

La compétitivité mondiale de l'industrie anime la croissance interne

La construction mécanique est tirée par la demande intérieure

Les entreprises investissent pour étendre leurs capacités

Fin de la crise du BTP 
L'Allemagne a surmonté une partie de ses problèmes structurels

La croissance se répercute maintenant sur l'emploi

La baisse des prélèvements sociaux est en cours sa part, bénéficie d'effets de calendrier : les ménages ont concentré leurs commandes sur la fin 2005 pour anticiper l'abandon des primes d'accession à la propriété, et ils anticipent pareillement à l'heure actuelle la hausse de la TVA.

La renaissance du BTP contribue ainsi à élargir la base de l'économie allemande : jusqu'ici, sa crise réduisait le taux de croissance d'au moins un quart de point. Le regain des investissements marque la fin de l'attentisme déclenché par le basculement à l'euro qui s'était traduit notamment par des taux d'intérêt plus élevés et une pratique plus restrictive du crédit bancaire ; depuis, les entreprises ont rationalisé leur production, bénéficiant dans le même temps d'une modération salariale unique en Europe. Dans ce contexte, la récente hausse des taux directeurs de la BCE n'aura pas d'effets sensibles sur l'évolution conjoncturelle, expliquent les milieux financiers. La stabilité des fondamentaux de son économie dans un environnement mondial et européen compétitif, le cap de réformes poursuivi ces dernières années, ont permis à l'Allemagne de surmonter plusieurs difficultés structurelles à l'origine de sa faible croissance depuis le début du siècle.

Et pour la première fois, la croissance atteint le marché de l'emploi. La tendance est ténue encore, mais depuis un an, le chômage baisse : le taux de chômage (critères OIT) est passé de $9,6 \%$ à $8,1 \%$ (Destatis). Le nombre des actifs occupés a augmenté de 310000 personnes ; le nombre des emplois soumis à cotisations sociales s'est accru de 50000 - la première hausse depuis cinq ans. Les entreprises recommencent à embaucher, comme le révèle le secteur du travail intérimaire : dans le tertiaire (transport, logistique et communication en tête), la demande augmente fortement, et commence à se heurter à une pénurie de main d'œuvre qualifiée. A l'inverse, le secteur des bas salaires, seul susceptible d'absorber les moins qualifiés parmi lesquels se recrute un tiers des chômeurs enregistrés par l'Agence fédérale pour l'emploi (4,45 millions), reste verrouillé. Son ouverture est l'un des prochains axes de réforme du gouvernement fédéral.

Pour inciter les entreprises à embaucher, les partenaires sociaux poursuivent leur politique de modération salariale, allant jusqu'à multiplier les accords particuliers. De son côté, le gouvernement fédéral tente de réduire les coûts salariaux annexes qui renchérissent le facteur travail outre-Rhin. La réforme de l'assurance maladie qui vient d'être adoptée (elle entrera en vigueur en 2009) s'inscrit dans ce contexte, bien qu'elle fasse l'unanimité contre elle. La hausse de la TVA poursuit le même objectif : les trois points de plus sont destinés à abaisser les cotisations. La réforme en cours de la fiscalité est destinée à améliorer le contexte réservé outre-Rhin à des entreprises en quête de compétitivité sur les marchés communautaire et mondial : mais elle a été ajournée du fait de la trop grande diversité encore des approches au sein de la coalition.

ENFIN, POUR LA PREMIERE FOIS DEPUIS 2001, l'Allemagne respectera cette année l'un des critères de Maastricht : le déficit sera vraisemblablement ramené à quelque 2,5\% selon le ministère fédéral des Finances ; il pourrait être nettement inférieur encore en 2007 et atteindre $1,9 \%$, à en croire les experts de l'IfW de Kiel. II faut voir dans cette réduction avant tout l'effet de la croissance : les recettes fiscales des collectivités territoriales ont crû de $+8,7 \%$ au premier semestre (Destatis), les redevances (dont surtout le péage dû par les transporteurs routiers) ont augmenté de 7,0\%. Quant à la hausse des recettes de l'assurance sociale $(+6,2 \%)$, elle s'explique uniquement par une modification de la date de versement des charges sociales, perçues depuis le début de l'année non plus à la fin, mais au début du mois. La situation financière du système de protection sociale demeure toujours aussi tendue.

L'amélioration de la situation budgétaire allemande n'est guère l'effet d'une réduction des dépenses : elle a été de $-1,3 \%$ seulement pour l'ensemble des collectivités territoriales. C'est le Bund qui a été le plus vertueux, avec -3,6\% (Länder : -0,5\%) ; les dépenses communales ont augmenté, elles, de $+2,7 \%$ (Destatis). Si le déficit s'est ainsi réduit conjoncturellement, reste à consolider structurellement les finances publiques. $A$ considérer la prudence avec laquelle Peer Steinbrück évoque sa cagnotte fiscale, on peut s'attendre à ce qu'il engage prochainement ce chantier. Mais la coalition craint de brider la dynamique de croissance retrouvée par une politique trop restrictive, comme ce fut partiellement le cas de Hans Eichel au début de son mandat, et d'étouffer le frémissement d'une consommation des ménages mise à mal par une facture énergétique en hausse et une faible croissance des revenus. Pourtant, la consolidation reste la condition sine qua non pour reconquérir durablement la confiance des acteurs économiques, entreprises et ménages. Déjà, Bert Rürup, président du Conseil des Sages qui remettra au gouvernement son rapport d'évaluation en décembre, rappelle à l'ordre la coalition (Handelsblatt, 05-09-06) : " II faut d'urgence une réduction drastique des dépenses. Une telle mesure est plus durable qu'une hausse de la fiscalité ».

Isabelle Bourgeois (10-10-2006) 